\title{
ROSAT - AN ALL-SKY X-RAY AND EUV SURVEY OF WHITE DWARFS
}

\author{
M.A.Barstow \\ X-ray Astronomy Group, Physics Department, University of Leicester \\ University Road,Leicester, LE1 7RH, UK
}

\begin{abstract}
The West German astronomy satellite ROSAT comprises two imaging telescopes - an X-ray instrument covering the energy band $0.15-2 \mathrm{keV}$ and an EUV instrument (provided by the UK) covering the range 0.02-0.21keV. A primary aim of the mission is to perform a sensitive all-sky survey with both telescopes (two 'colours' in the EUV) in the first 6 months. This will be followed by a least one year of pointed observations at specific targets. This paper briefly describes the ROSAT instrumentation and discusses the likely impact of the survey on white dwarf studies.
\end{abstract}

\section{INTRODUCTION}

ROSAT is a West German X-ray astronomy mission led by the Max Planck Institut für Extraterrestriche Physik. The satellite comprises two instruments, a large Wolter I imaging X-ray telescope (XRT) covering an energy range $0.15-2 \mathrm{keV}(80-6 \AA)$ and a wide field EUV telescope spanning the band $\approx 0.02-0.21 \mathrm{keV}(620-60 \AA)$. The latter instrument, the Wide Field Camera (WFC), is being provided by the UK. The US will provide a high resolution imaging detector (HRI) for the XRT focal plane (in addition to the German imaging proportional counter - PSPC) and the launch, on a Delta rocket in early 1990. The major objective of the mission is to perform the first imaging all-sky surveys in both X-ray and EUV bands during the initial six months of operation. After this a minimum of one year of pointed observations are planned, with possible extensions for up to two years depending on the lifetime of the satellite. During the survey the XRT will employ the energy sensitive PSPC at its focus and the WFC will image the sky in two wavebands by use of selected filters. Two more filters will be available to extend the WFC spectral coverage to lower energies during the pointed observations. Experience with the Einstein and EXOSAT satellites has shown how important soft $\mathrm{X}$-ray observations are in the study of hot white dwarfs (WDs) (eg. Paerels et al., 1987; Petre et al., 1986) particularly in determining the concentrations of trace elements such as He and CNO (eg. Paerels et al., 1987; Barstow, 1988). A sensitive all-sky survey in soft X-ray and EUV bands will provide an unprecedented database with which to pursue these studies. This paper gives brief descriptions of the XRT and WFC instruments and discusses their sensitivities in the context of white dwarf observations.

\section{DESCRIPTION OF THE XRT AND WFC}

Both these instruments have been described in detail in a number of papers (eg. Trumper, 1984: XRT; Barstow and Pounds, 1988: WFC). Consequently, this discussion is restricted to the important details and scientific performance.

The XRT consists of a nest of four Wolter I grazing incidence telescopes having an intrinsic resolution of a few ", with a focal plane assembly of two PSPCs and one HRI which are mounted on a carousel. The PSPC (Pfefferman et al, 1986) has a relatively large $2^{\circ}$ field of view with an on-axis resolution of $30^{\prime \prime}$ FWHM and an average over the field of view of 2 '. Its nominal energy range is $0.1-2 \mathrm{keV}(6-80 \AA)$ and the spectral resolution of $<45 \% \mathrm{FWHM}$ at $1 \mathrm{keV}$ allows four distinguishable 'colour' bands. The HRI (Pfefferman et al, 1986) is a microchannel plate (MCP) detector, 
similar to that flown on Einstein but with a CsI photocathode to enhance the quantum efficiency. It has a resolution of 1.7" FWHM but poor energy resolution. A filter of aluminised Parylene N protects the detector from geocoronal radiation and ions.

The WFC has a nest of three Wolter-Schwarzchild type I mirrors with two identical MCP detectors mounted on a turret mechanism in the focal plane. The mirror grazing angles chosen $\left(\approx 7.5^{\circ}\right)$ allow the collecting area to be optimised while retaining a wide $\left(5.0^{\circ} \mathrm{diam}\right.$.) field of view and a low energy reflectivity cutoff at $0.21 \mathrm{keV}(60 \AA)$. An onaxis resolution of $2.3^{\prime \prime}$ HEW is expected degrading to $4.4^{\prime \prime}$ HEW at the edge of the field as a result of inherent optical aberrations. Hence, the average survey resolution will be $3.5^{\prime \prime} \mathrm{HEW}$. The MCP detector has a CsI photocathode to enhance its quantum efficiency but has no intrinsic energy resolution. A filter wheel assembly mounted near the focal plane contains a number of filters which can be selected to define wavelength pass bands, suppress geocoronal radiation and prevent detection of $\mathrm{UV}$ radiation from hot $\mathrm{O} / \mathrm{B} 0$ stars.

The XRT and WFC spectral bands and respective sensitivities are summarised in table I. In the context of WD studies the PSPC can be taken to have a single energy band (44-80 $\AA$ ) since in general no significant flux is observed below $44 \AA$ and the PSPC energy resolution is poorest in this region.

\section{OBSERVING HOT WHITE DWARFS}

Our current understanding of the formation and evolution of WDs has recently been reviewed by Sion (1986). It is clear that many problems concerning the relationships between different groups of objects remain to be understood, in particular between H-rich and He-rich WDs and their progenitors. There is evidence that He-rich WDs contain significant quantities of $\mathrm{CNO}$ but their abundances are not well determined. The combination of ROSAT XRT and WFC is an ideal tool for such studies. Figure 1 illustrates this, comparing the instrument bands ( 3 in the survey and up to 5 in pointed mode) with some typical WD spectral models. This indicates that the instruments are much more sensitive to $\mathrm{H}$ dominated stars than $\mathrm{He}$-rich ones, as might be expected given the relatively high opacity of $\mathrm{He}$ at soft $\mathrm{X}$-ray energies. The ability of ROSAT to observe WDs has been quantified by convolving model WD spectra with the instrument response for each bandpass. A minimum detectable temperature limit can be estimated for each bandpass, as a function of absorbing column density, as illustrated in figure 2 for pure $H$ and pure He atmospheres.

To assess the potential of the sky survey requires an estimate of the number of WDs that we might expect to see. Fleming, Liebert and Green (1986; hereafter FLG) present a summary of space densities for DA and DO/DB WDs subdivided by temperature. Their estimated scale height is $250 \mathrm{pc}$. If the volume accessible to ROSAT is known for each band, calculating the number of stars is simple. However, this depends on assumptions made about the ISM and WD radii. A good indication of the effect of the ISM can be obtained by assuming that it is uniform with a mean density of 0.07 atoms $\mathrm{cm}^{-3}$, although in reality there are large line of sight variations with viewing direction (eg. Paresce, 1984). Based on this assumption the maximum distances at which WDs could be detected were determined for each bandpass as a function of temperature. WD radii were assumed to be $0.0125 R_{O}$. These distances and the respective column densities are summarised in table II for the temperatures corresponding to the centre of the ranges for which FLG tabulate space densities. To account for WDs in binary systems their densities are multiplied by a factor of 2 in this calculation. The expected numbers of DA WDs in the volumes defined are also listed in table II. It is not possible to perform a similar calculation for DO/DB WDs. The temperatures to which $R O S A T$ is sensitive are 
TABLE I. ROSAT XRT+PSPC \& WFC Filters - Wavebands and Sensitivity

\begin{tabular}{|c|c|c|c|c|c|c|c|}
\hline Instrument & $\begin{array}{l}\text { Detector } \\
\text { or } \\
\text { Filter Type [a] }\end{array}$ & $\begin{array}{l}\text { Survey (S) } \\
\text { or } \\
\text { Pointed (P) }\end{array}$ & $\begin{array}{l}\text { FOV } \\
\text { Diam. } \\
\text { (deg.) }\end{array}$ & $\begin{array}{l}\text { 'Mean' } \\
\text { Wavelength } \\
(\AA)\end{array}$ & $\begin{array}{l}\text { Bandpass }(\AA) \\
\text { (at } 10 \% \text { of peak } \\
\text { efficiency) }\end{array}$ & $\begin{array}{l}\text { Point } \\
\text { Sensit } \\
(\mu \mathrm{J} y)\end{array}$ & $\begin{array}{l}\text { vity[b] } \\
\left(\mathrm{HZ} 43^{-1}\right)\end{array}$ \\
\hline$\overline{X R T}$ & PSPC & $\mathbf{S}+\mathbf{P}$ & 2.0 & 60 & $44-80$ & 0.47 & 6000 \\
\hline \multirow[t]{4}{*}{ WFC } & C/Lexan/B $(\times 2)$ & $S+P$ & 5 & 100 & $60-140$ & 1.0 & 2400 \\
\hline & $\mathrm{Be} / \operatorname{Lexan}(\times 2)$ & $S+P$ & 5 & 140 & $112-200$ & 1.4 & 4800 \\
\hline & $\mathrm{Al} /$ Lexan & $\mathbf{P}$ & 2.5 & 180 & $150-220$ & 7.3 & 1300 \\
\hline & $\mathrm{Sn} / \mathrm{Al}$ & $\mathrm{P}$ & 2.5 & 600 & $530-720$ & 220 & 160 \\
\hline
\end{tabular}

[a] Provisional. [b] For $5 \sigma$ significance, exposure time of 2000s (a typical value for each filter for the survey and for pointed observations) and 'typical' background. The right hand column expresses the sensitivity in terms of the flux from the white dwarf HZ43, the brightest known EUV source.

Figure 1. White dwarf model atmospheres for a $60000 \mathrm{~K}$ star comprising [1] pure $\mathrm{H},[2] \mathrm{He} / \mathrm{H}=10^{-4}$ and [3] pure $\mathrm{He}$. The

Figure 2. The minimum WD temperature to which solid vertical lines indicate the mean wavelengths of each band. photon energy and wavelength
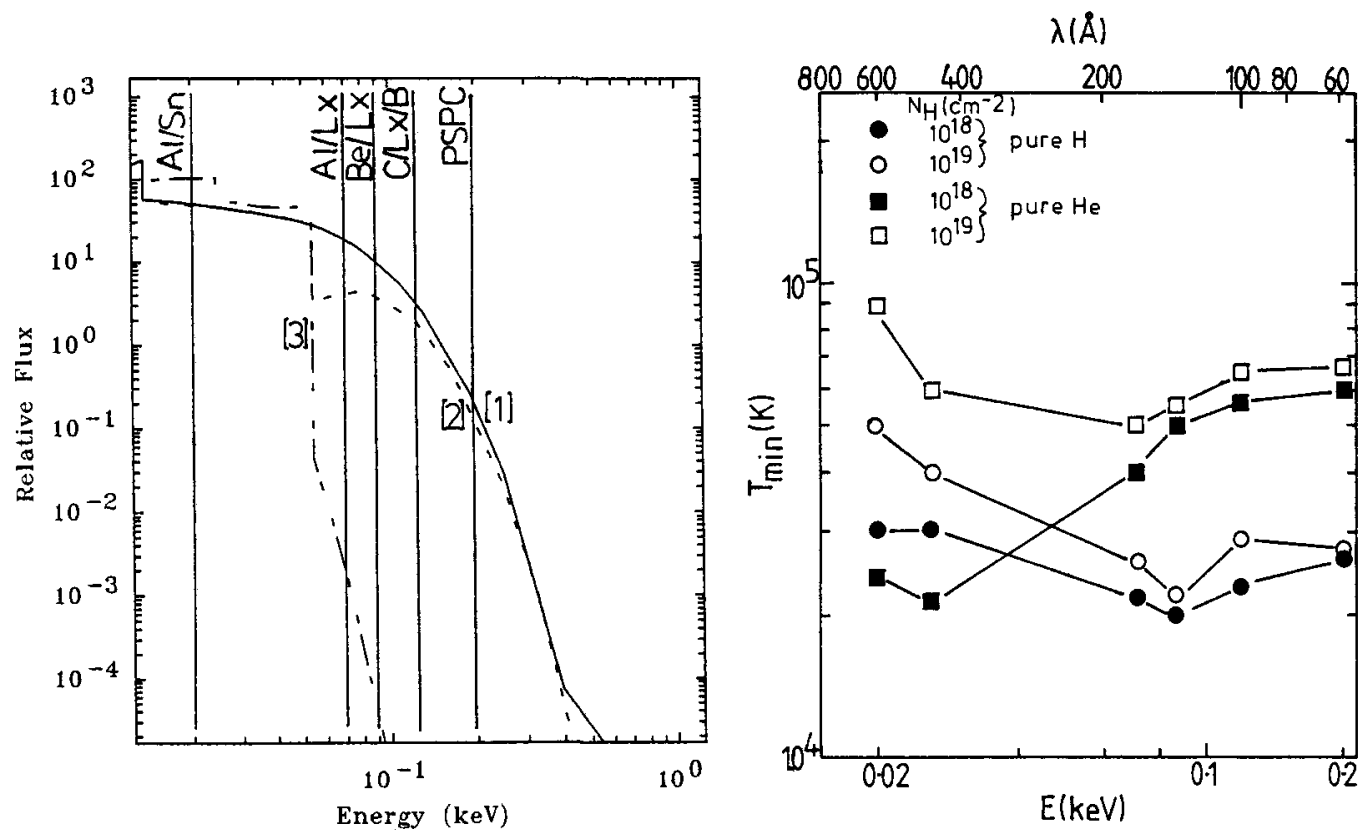

TABLE II. Numbers of DA WDs detected in each ROSAT band with maximum distances and values of $\mathrm{N}_{\mathrm{H}}^{[\mathrm{a}]}$.

\begin{tabular}{lllllll}
\hline & & XRT & & WFC & & \\
& & PSPC & C/Lexan/B & Be/Lexan & Al/Lexan & Al/Sn \\
\hline $30000 \mathrm{~K}$ & $\mathrm{~d}(\mathrm{pc})$ & 175 & 78 & 104 & 55 & $<30$ \\
& $\mathrm{~N}_{\mathrm{H}}\left(\mathrm{cm}^{-2}\right)$ & $3.6 \times 10^{19}$ & $1.6 \times 10^{19}$ & $2.2 \times 10^{19}$ & $1.0 \times 10^{19}$ & $<10^{18}$ \\
$60000 \mathrm{~K}$ & $\mathrm{~d}(\mathrm{pc})$ & 1240 & 554 & 350 & 175 & 40 \\
& $\mathrm{~N}_{\mathrm{H}}\left(\mathrm{cm}^{-2}\right)$ & $2.6 \times 10^{20}$ & $1.0 \times 10^{20}$ & $7.4 \times 10^{19}$ & $3.7 \times 10^{19}$ & $8.0 \times 10^{18}$ \\
No of DA WDs & 5500 & 2200 & 1400 & 200 & $<10$ \\
\hline
\end{tabular}

[a] For ISM with constant volume number density of neutral hydrogen of $0.07 \mathrm{~cm}^{-3}$ (Paresce 1984). 
generally in the upper range considered by FLG, where the space density is very low. Within the volumes accessible very few stars would be seen. However, it is known that hot DO stars exist with temperatures substantially in excess of $80000 \mathrm{~K}$ (FLG's upper limit). ROSAT could see such objects up to distances of $1 \mathrm{kpc}$ and $\mathrm{N}_{\mathrm{H}}$ values of $2.0 \times 10^{20} \mathrm{~cm}^{-2}$.

\section{CONCLUSION}

The survey bands of ROSAT are capable of detecting hot WDs at distances of more than $1 \mathrm{kpc}$ and column densities above $1-2 \times 10^{20} \mathrm{~cm}^{-2}$. It is expected that $\approx 5500, \approx 2200$ and $\approx 1400 \mathrm{DA}$ WDs will be detected in PSPC, C/Lexan/B and $\mathrm{Be} /$ Lexan bands (the survey bands) respectively, but large uncertainties are present in these estimates. However, resolving problems like these is an important reason for performing the survey. Approximately 1500 WDs are known to exist of which $\approx 30 \%$ (about 450 ) have temperatures $>20000 \mathrm{~K}$. Clearly most of those WDs detected by ROSAT will be unidentified and a considerable follow-up programme of optical observations will be needed. Realistic estimates of the numbers of detectable He-rich WDs cannot be made with current data. An all-sky survey will be of particular importance in population studies of these objects as ROSAT is very sensitive to those temperature ranges not easily studied by optical or UV observations.

Observations of a few WDs with Einstein and EXOSAT has shown what information can be obtained (eg. $\mathrm{T}_{\text {eff }}, \mathrm{N}_{\mathrm{H}}$ and $\mathrm{H} / \mathrm{He}$ ratio) from soft $\mathrm{X}$-ray photometry. The ROSAT sky survey will represent a huge increase in the statistical sample available for population studies, yielding an accurate luminosity function and the relationship between He abundances and $T_{\text {eff }}$.

\section{ACKNOWLEDGEMENTS}

ROSAT is a West German mission, led by MPE to which the UK has contributed the WFC. I would like to thank the project scientist Prof. J.Trümper for allowing me to discuss the XRT performance in this paper. The WFC is supported by the SERC, UK and is being constructed by a consortium comprising - Leicester University, Birmingham University, Rutherford-Appleton Laboratory, Mullard Space Science Laboratory and Imperial College, London.

\section{REFERENCES}

Barstow,M.A., 1988, these proceedings.

Barstow,M.A. and Pounds,K.A., 1988, proceedings of the NATO ASI, 'Hot Thin Plasmas in Astrophysics', ed. R.Pallavicini, 359.

Fleming,T., Liebert,J. and Green,R.F., 1986, Astrophys.J., 308, 176.

Paerels,F.B.S., Heise,J., Kahn,S.M. and Rodgers,R.D., 1986, Astrophys.J., 322, 315.

Paresce,F., 1984, Astron.J., 89, 1022.

Petre,R., Shipman,H.L. and Canizares,C.R., 1986, Astrophys.J., 304, 356.

Pfefferman,E.,et al., 1986, Proc SPIE, 733, 519.

Sion,E.M., 1986, Publ.astr.Soc.Pac., 98, 821.

Trümper,J., 1984, Physica Scripta, T7, 209. 